\title{
Pulmonary paracoccidioidomycosis: a case report of reactivation in a patient receiving biological therapy
}

\author{
Lúcia Carla Polaco Covre ${ }^{[1]}$, Pâmela Mazzini Hombre ${ }^{[1]}$, Aloísio Falqueto ${ }^{[2],}$ \\ Paulo Mendes Peçanha ${ }^{[2]}$ and Valéria Valim ${ }^{[3]}$
}

\begin{abstract}
[1]. Curso de Medicina, Hospital Universitário Cassiano Antônio Moraes, Universidade Federal do Espírito Santo, Vitória, ES, Brasil. [2]. Ambulatório de Doenças Infecciosas e Parasitárias, Hospital Universitário Cassiano Antônio Moraes, Universidade Federal do Espírito Santo, Vitória, ES, Brasil. [3]. Ambulatório de Reumatologia, Departamento de Clínica Médica, Hospital Universitário Cassiano Antônio Moraes, Universidade Federal do Espírito Santo, Vitória, ES, Brasil.
\end{abstract}

\begin{abstract}
Paracoccidioidomycosis is an endemic disease in Latin America that is rarely associated with immunosuppression and biological therapy. Herein, we report for the first time a case of pulmonary paracoccidioidomycosis reactivation after infliximab treatment. A 47-year-old man from Brazil received infliximab to treat psoriatic spondyloarthropathy and presented with cough, dyspnea, weight loss, and fever. Chest computed tomography revealed a pulmonary nodule and biopsy confirmed paracoccidioidomycosis. Treatment with sulfamethoxazole and trimethoprim was initiated for fungal infection and infliximab was reintroduced two months later. Considering his clinical improvement and favorable radiologic evolution, antifungal therapy was discontinued after 29 months.
\end{abstract}

Keywords: Paracoccidioidomycosis. Infliximab. Immunosuppression.

\section{INTRODUCTION}

Paracoccidioidomycosis (PCM) is a systemic mycosis with dragged evolution caused by the fungus Paracoccidioides sp., which is endemic in regions of Latin America. The infection occurs through inhalation of the fungus during activities related to soil management. Infected individuals can remain asymptomatic or develop acute PCM. Additionally, throughout life, quiescent lesions may be reactivated (a clinical condition known as the chronic adult form) or the residual form may develop, mainly characterized by the formation of fibrotic sequelae ${ }^{1-3}$. Smoking and alcoholism behaviors have been associated as potential factors for PCM reactivation ${ }^{1-3}$. In addition, immunosuppressive diseases or medications may also play a role in disease progression ${ }^{4,5}$.

Anti-tumor necrosis factor alpha (anti-TNF- $\alpha$ ) inhibitors are drugs administered to treat auto-immune and auto-inflammatory diseases. These drugs are now widely administered to effectively reduce inflammation ${ }^{4-7}$. On the other hand, the use of antiTNF- $\alpha$ therapy has been associated with an increased risk of severe opportunistic infections, as well as tuberculosis and

Corresponding author: $\mathrm{Dr}^{\mathrm{a}}$. Valéria Valim.

e-mail: val.valim@gmail.com

Received 8 June 2017

Accepted 22 December 2017 histoplasmosis. In addition, infections such as aspergillosis, candidiasis, bartonellosis, legionellosis, listeriosis, leprosy, non-tuberculous mycobacterial infections, and others have been associated with anti-TNF- $\alpha^{5-7}$.

Despite the few reports describing the association between biological therapy and reactivation of PCM, none of them described a correlation between the use of infliximab to reactivation of the disease ${ }^{4,8-10}$. Thus, this report presents a case of pulmonary PCM reactivation in a patient receiving infliximab therapy.

\section{CASE REPORT}

A 47-year-old man from Espírito Santo, Southeastern Brazil, who was a non-smoker and non-alcoholic with a past history of engaging in soil-related activities, presented with recurrent bilateral uveitis since 2007, associated with lower back pain, calcaneodynia, and inflammatory cutaneous lesions in the knees and elbows, consistent with signs of psoriasis. In 2010, he was diagnosed with psoriatic spondyloarthritis. He was initially administered methotrexate ( $20 \mathrm{mg} / \mathrm{week})$ after negative screening results for tuberculosis (negative tuberculin skin test results and normal chest radiography). After four months, the treatment was suspended due to gastrointestinal intolerance and remission of disease symptoms.

However, in 2013, he presented uveitis reactivation with a reduction in visual acuity and associated arthritis in the knee. He received a new cycle of prednisone, and azathioprine 
administration was initiated $(2 \mathrm{mg} / \mathrm{kg} /$ day $)$, followed by a gradual reduction of the systemic corticosteroid. Azathioprine was substituted by infliximab after 20 days because of the development of febrile neutropenia.

After new negative tuberculosis screening results (negative tuberculin skin test results and normal chest radiography), 11 infusions of infliximab $(0.5 \mathrm{mg} / \mathrm{kg})$ were administered for 18 months. Nevertheless, in December 2014, the patient presented with dry cough, dyspnea, arthralgia, weight loss, fever, and sweating. On examination, he was pallid and eupneic, and exhibited decreased breath sounds in the lung bases and sparse rales without cardiovascular or abdominal changes. Chest computed tomography (CT) showed a nodule with soft-tissue density of undetermined nature in the lower lobe of the left lung associated with mediastinal and perihilar lymphadenopathy, and cortical micronodules, all smaller than $5 \mathrm{~mm}$, which were more numerous in the left lung (Figure 1).

Mediastinoscopy with lymph node biopsy was inconclusive and showed negative results for acid-alcohol-resistant bacillus (BAAR) and fungi. Infliximab treatment was suspended until the definition of the pulmonary condition was fulfilled. After suspension of the immunosuppressive therapy, the patient progressed with general clinical and respiratory improvements. However, chest CT still showed a nodule with soft-tissue density in the lower lobe of the left lung measuring approximately $10 \mathrm{~mm}$ and perihilar ganglia calcifications. Thus, in 2015, segmentectomy of the left inferior pulmonary lobe (pulmonary nodule site) and periaortic lymph node biopsy were performed. Histopathologic results of segmentectomy revealed chronic granulomatous inflammation, with rounded structures of varying sizes and lateral budding forms compatible with those of Paracoccidioides sp. (Figure 2). The mediastinal lymph node biopsy showed chronic granulomatous inflammation in areas of central fibrosclerosis and negative results for BAAR and fungi.

The treatment for PCM was initiated with $800 \mathrm{mg}$ of sulfamethoxazole plus $160 \mathrm{mg}$ of trimethoprim twice a day. Detection of anti-Paracoccidioides brasiliensis, and antiAspergillus and anti-Histoplasma antibodies, using the double immunodiffusion method, was negative at the beginning of the treatment and during the follow-up. After two months, due to favorable clinical evolution, infliximab was reintroduced $(5 \mathrm{mg} / \mathrm{kg}$ every six weeks). After 29 months of PCM treatment, new control chest CT showed a fibrous and calcified band in the lower lobe of the left lung besides persisting calcified hilar ganglia and mediastinal lymphadenopathy. Based on the residual aspect of the lesions observed using control $\mathrm{CT}$ and clinical improvement of the patient, the treatment for PCM was discontinued and clinical follow-up was maintained. Currently, the patient is undergoing regular treatment for psoriatic arthritis with infliximab.

\section{DISCUSSION}

TNF- $\alpha$ is a pro-inflammatory cytokine critical for the formation and maintenance of the granuloma. This cytokine also mediates immune protection against intracellular pathogens and has a central role in the pathogenesis of auto-immune and

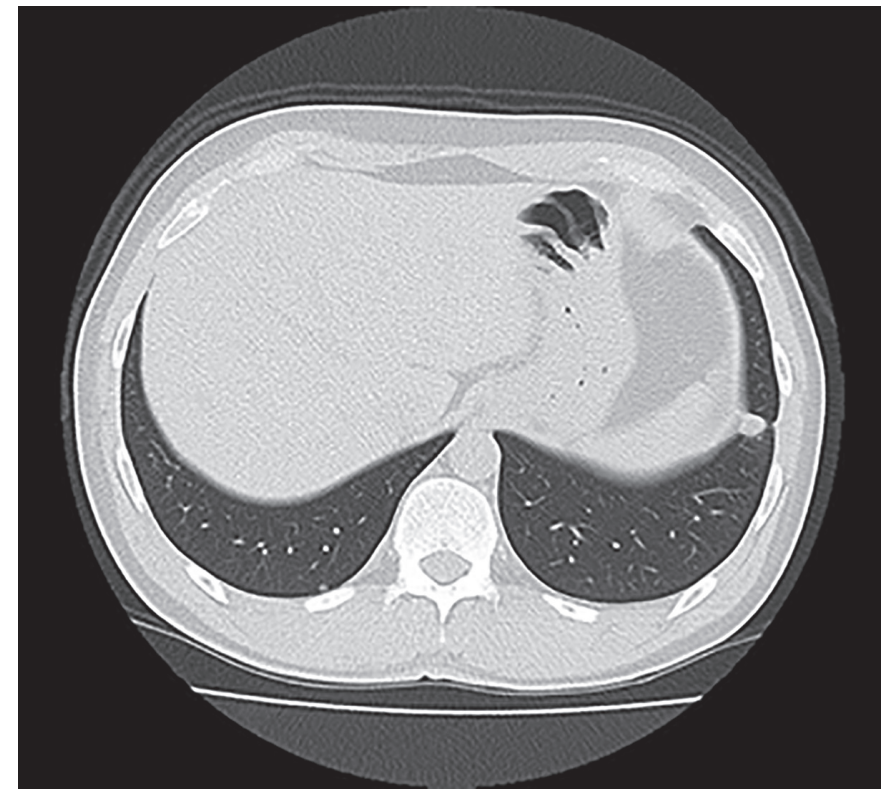

FIGURE 1: Chest computed tomographic scan. Axial section showing a nodule with soft-tissue density in the lower lobe of the left lung measuring approximately $10 \mathrm{~mm}$.

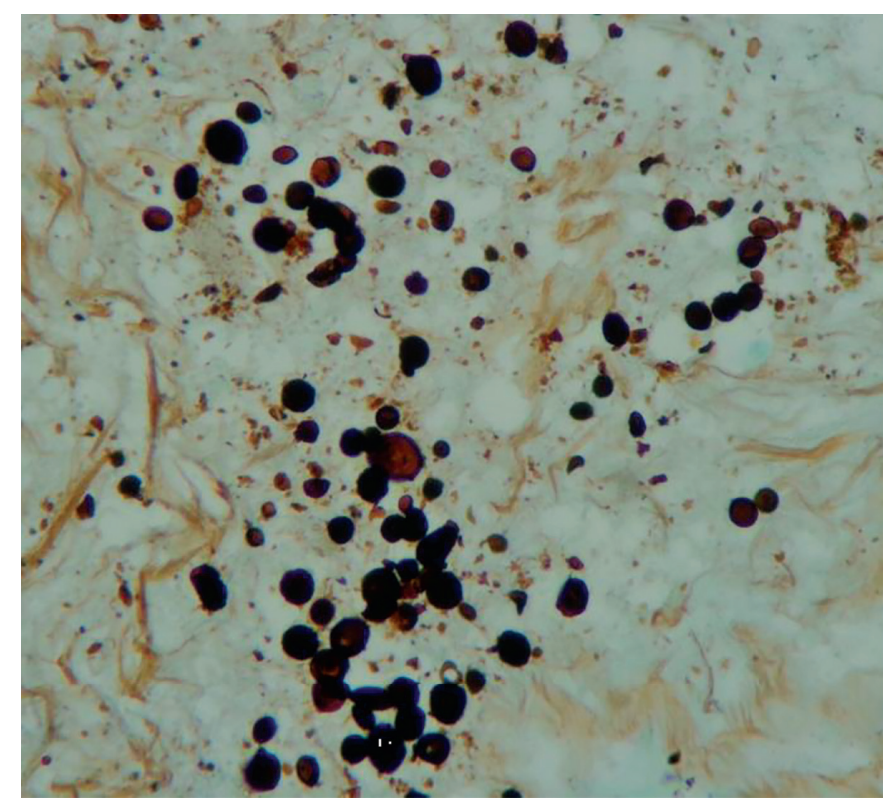

FIGURE 2: Left lung (lower lobe) excisional biopsy: Chronic granulomatous inflammation and presence of rounded structures of variable size, with lateral budding forms compatible with Paracoccidioides sp. negative acid-alcoholresistant bacillus results. Histological analysis revealed compatibility with paracoccidioidomycosis.

auto-inflammatory diseases. Therefore, the administration of anti-TNF- $\alpha$ therapy has been shown to be an effective tool to treat these diseases ${ }^{7}$.

However, TNF- $\alpha$ inhibitors have been associated with an increased risk of developing opportunistic infections ${ }^{4-7}$. A systematic review and meta-analysis involving 5,014 randomized 
TABLE 1: Case review of associations between PCM and the use of immunosuppressive drugs between 1976 and 2016.

\begin{tabular}{|c|c|c|c|}
\hline Sex/age (years) & Associated disease & Immunosuppressive drug(s) & Reference \\
\hline$M / 56$ & Temporal arteritis & Corticosteroids & 4 \\
\hline $\mathrm{M} / 55$ & Oat cell carcinoma (mediastinum) & Cyclophosphamide, Vincristine, and Adriamycin & 4 \\
\hline $\mathrm{M} / 48$ & Hodgkin's disease & Methotrexate, Vincristine, Procarbazine, and Prednisone & 4 \\
\hline $\mathrm{F} / 34$ & Systemic lupus erythematosus & Prednisone & 4 \\
\hline $\mathrm{F} / 29$ & Renal transplantation & Azathioprine and Prednisone & 4 \\
\hline $\mathrm{M} / 43$ & Renal transplantation & Azathioprine, Cyclosporine, and Prednisone & 4 \\
\hline$M / 66$ & Renal transplantation & Prednisone, Mesalazine, and Tacrolimus & 8 \\
\hline $\mathrm{M} / 47$ & Psoriatic spondyloarthropathy & Methotrexate, Azathioprine, Prednisone, and Infliximab & Present report \\
\hline
\end{tabular}

PCM: paracoccidioidomycosis; M: male; F: female. *Extra-pulmonary involvement (cervical tumor). All other cases involved pulmonary involvement. Source: Review of the authors' table from Woyciechowsky TG et al (ref. 4).

patients who received infliximab, adalimumab, or placebo concluded that anti-TNF- $\alpha$ therapy increases the risk of infection when compared with placebo (odds ratio $2.0 ; 95 \%$ confidence interval 1.3-3.1 $)^{11}$.

The association between PCM and the use of immunosuppressive drugs has already been described in the literature, but until now, there have been no reports on disease reactivation after infliximab administration. Most of the 11 cases previously described were in men aged older than 40 years with pulmonary involvement (Table 1) 1,8-10 $^{\text {. }}$

The reported case is suggestive of PCM's chronic form due to the pattern of nodular and micronodular lesions involving both lungs. Brazil has the largest number of PCM cases in Latin America, and southeast Brazil, including Espírito Santo State, is considered a high endemic area ${ }^{12}$. Despite this, there is insufficient PCM screening, enabling the reactivation of latent infections after immunosuppression therapy.

Currently, the interruption of anti-TNF- $\alpha$ therapy is proposed if a fungal infection has been diagnosed during treatment ${ }^{6}$ In this case report, the only associated risk factor for disease reactivation was the administration of immunosuppressive drugs: mainly the association of infliximab and prednisone. These were discontinued when the definition of the pulmonary condition was fulfilled and reintroduced after clinical and radiologic improvement with antifungal therapy. Although there are no clinical protocols to support the management of these patients, the conduct described here allowed for successful outcomes.

The reported case warns about the importance of suspecting PCM in patients with respiratory symptoms undergoing biological therapy, mainly in those from endemic areas.

\section{Acknowledgments}

We offer our deepest thanks to the institutions that provided technical support for the development and implementation of this study.

\section{Conflict of interest}

The authors declare that there is no conflict of interest.

\section{Financial support}

This research did not receive any specific grant from funding agencies in the public, commercial, or not-for-profit sectors.

\section{REFERENCES}

1. Restrepo A, Gómez BL, Tobón A. Paracoccidioidomycosis: Latin America's own fungal disorder. Curr Fungal Infect Rep. 2012;6(4):303-11.

2. Marques SA. Paracoccidioidomycosis: epidemiological, clinical, diagnostic and treatment up-dating. An Bras Dermatol. 2013;88(5):700-11.

3. Santos WA, Silva BM, Passos ED, Zandonade E, Falqueto A. Associação entre tabagismo e paracoccidioidomicose: um estudo de caso-controle no Estado do Espírito Santo, Brasil. Cad Saúde Pública. 2003;19(1):245-53.

4. Woyciechowsky TG, Dalcin DC, Santos JWA, Michel GT. Paracoccidioidomycosis induced by immunosuppressive drugs in a patient with rheumatoid arthritis and bone sarcoma: case report and review of the literature. Mycophatologia. 2011;172(1):77-81.

5. Kopylov U, Afif W. Risk of infections with biological agents. Gastroenterol Clin North Am. 2014;43(3):509-24.

6. Smith JA, Kauffman CA. Endemic fungal infections in patients receiving tumor necrosis factor- $\alpha$ inhibitor therapy. Drugs. 2009;69(11):1403-15. 
7. Johnston BL, Conly JM. Tumour necrosis factor inhibitors and infection: what is there to know for infectious diseases physicians? Can J Infect Dis Med Microbiol. 2006;17(4):209-12.

8. Goes HFO, Durães SMB, Lima CS, Souza MB, Vilar EAG, Dalston MO. Paracoccidioidomycosis in a renal transplant recipient. Rev Inst Med Trop Sao Paulo. 2016;58(12)1-3.

9. Pontes AM, Borborema J, Correia CRB, de Almeida WL, Maciel RF. A rare paracoccidioidomycosis diagnosis in a kidney transplant receptor: case report. Transplant Proc. 2015;47(4):1048-50.

10. Von Glehn F, Damasceno A, Miotto N, Naseri EP, Costallat LTL, França MC, et al. Carpal tunnel syndrome with paracoccidioidomycosis. Emerg Infect Dis. 2012;18(8):1390-92.
11. Bongartz T, Sutton AJ, Sweeting MJ, Buchan I, Matteson EL, Montori V. Anti-TNF antibody therapy in rheumatoid arthritis and the risk of serious infections and malignancies: systematic review and meta-analysis of rare harmful effects in randomized controlled trials. JAMA. 2006;295(19):2275-85.

12. Peçanha PM, Batista Ferreira ME, Missaroni Peçanha MA, Schmidt EB, Lamas de Araújo M, Zanotti RL, et al. Paracoccidioidomycosis: epidemiological and clinical aspects in 546 cases studied in the State of Espírito Santo, Brazil. Am J Trop Med Hyg. 2017;97(3):836-44. 